\title{
Caracterización físico y sensorial de 8 variedades de la especie Phaseolus vulgaris $L$.
}

Physical and sensory characterization of 8 varieties of the species Phaseolus vulgaris $L$. Nicodemo Crescencio Jamanca Gonzales ,Juana Consuelo Aliaga Camarena

\section{RESUMEN}

Objetivo: Evaluar las características físicas y sensoriales de 8 variedades de frijol canario (Phaseolus vulgaris L.), cultivadas en la campaña 2016 - 2017 (agosto - diciembre) en el campo experimental, Los Anitos de la Universidad Nacional de Barranca. Métodos: Se evaluó la masa y volumen de 100 granos, contenido de testa, humedad, capacidad de absorción de agua, tiempo de cocción y evaluación sensorial del producto cocido empleando panelistas entrenados, para determinar su grado de aceptación por el consumidor. Resultados: La variedad Blanco Huanuqueño mostró mayor peso y volumen. El canario procedente de Ocros (Ancash) presentó el mayor contenido de humedad. Los porcentajes de testa oscilan entre 5,62-7,06\%. La variedad Laran Mejorado muestra menor valor de capacidad de absorción de agua. Las variedades de frijol presentaron tiempos de cocción que oscilaron entre 79 y 85 minutos. Conclusiones: Los resultados sensoriales demuestran que las variedades canario procedentes de Huacaybamba, Canario procedente de Ocros, Canario Centenario y Canario Línea 4, obtienen mayor aceptabilidad por el consumidor.

Palabras clave: Phaseolus vulgaris L., variedad, absorción de agua, tiempo de cocción, evaluación sensorial.

\section{ABSTRACT}

Objective: To evaluate the physical and sensory characteristics of 8 Canarian bean varieties (Phaseolus vulgaris L.), grown in the 2016 - 2017 campaign (August - December) in the experimental field, Los Anitos of the National University of Barranca. Methods: The mass and volume of 100 grains, testa content, humidity, water absorption capacity, cooking time and sensory evaluation of the cooked product were evaluated using trained panelists to determine their degree of acceptance by the consumer. Results: The Blanco Huanuqueño variety showed greater weight and volume. The canary from Ocros (Ancash) had the highest moisture content. The testa percentages range between $5.62-7.06 \%$. The Laran Mejorado variety shows lower value of water absorption capacity. The bean varieties presented cooking times that ranged between 79 and 85 min. Conclusions: The sensory results show that the Canary varieties from Huacaybamba, Canary from Ocros, Canary Centenary and Canary Line 4, obtain greater acceptability by the consumer.

Keywords: Phaseolus vulgaris L.; variety, water absorption, cooking time, sensory evaluation.

\footnotetext{
${ }^{1}$ Universidad Nacional de Barranca. Barranca, Perú.
} 


\section{INTRODUCCIÓN}

El frijol (Phaseolus vulgaris L.) de la variedad canario (amarillo) es una de las leguminosas que la población local, regional del Perú tiene como preferencia en su alimentación diaria y su consumo per cápita es $2,6 \mathrm{~kg}$ a nivel de Lima y 2,9 kg en el resto del país (INEI, 2009). En cuanto a nivel latinoamericano las cifras son relativamente bajas, comparadas al promedio de Brasil con 16,1 kg o al de México con $11 \mathrm{~kg}$ por persona en promedio anual (Dirección General de Industrias Básicas, 2012). Asimismo, las variedades del frijol se pueden clasificar de acuerdo a diversos criterios, como lo menciona Ulloa, Ulloa, Ramírez y Ulloa (2011), por su consumo como grano seco y como grano y vaina verde; además es común clasificarlas por su color como blanco, crema, amarillo, café marrón, rosado, rojo, morado, negro u otros.

Las caracterizaciones de variedades de frijol han sido desarrolladas con el interés de determinar su valor nutricional y comercial, como las desarrolladas por Jacinto y Campos (1993), que determinaron el efecto de la cocción sobre características tecnológicas y nutricionales de 7 variedades de frijol, encontrándose diferencias significativas entre variedades en el peso, tamaño, \% de testa, tiempo de cocción, actividad del inhibidor de tripsina y digestibilidad. Asimismo, en la evaluación de la calidad de la proteína de 4 variedades mejoradas de frijol desarrollado por Cárdenas. et al. (2000) al evaluar 4 variedades mejoradas y una variedad comercial de frijol, encontraron valores promedio del valor biológico y la razón proteica neta considerando el agua de remojo fue $55 \%$ y $2,9 \%$. En el estudio desarrollado por Mederos y Reynaldo (2007) se determinó indicadores de calidad de 11 genotipos de la especie Phaseolus vulgaris L., cuyas características físicas, estuvieron referidas al peso y volumen de 100 granos, los porcentajes de humedad y testa, asimismo, se evaluaron las características culinarias, representadas por la capacidad de absorción de agua y el tiempo de cocción, encontrándose diferencias entre las mejores variedades de grano rojo y negro.

El frijol constituye para la población de Barranca una de las principales fuentes de proteína, por el bajo costo de ésta en comparación con la proteína de origen animal, considerando que el frijol es una buena fuente de proteínas (20 -25\%) y carbohidratos (50 $60 \%$ ), como lo señala Carmona (2005). Sin embargo, presenta algunas limitantes, tales como su deficiencia en aminoácidos azufrados y la presencia de algunos factores antinutricionales (Jacinto y Campos, 1993).

El objetivo del presente estudio fue evaluar las características físicas como masa de 100 granos $(\mathrm{g})$, volumen de 100 granos $(\mathrm{ml})$, humedad del grano (\%), capacidad de absorción de agua (\%), testa (\%) y tiempo de cocción (min); en la estimación de atributos sensoriales se evaluó la consistencia del caldo, olor, sabor, textura y color, de 8 variedades de frijol, coleccionadas y producidas por la Universidad Nacional de Barranca, con la finalidad de apreciar la variedad de frijol sobresale en sus características físicas y sensoriales posterior a la cocción.

\section{MATERIAL Y MÉTODOS}

La presente investigación según su propósito es de tipo aplicada, en tanto por la dirección en el tiempo, es de corte transversal. Asimismo, por el nivel de capacidad explicativa de la investigación es descriptivo, con enfoque cuantitativo. La investigación se realizó en febrero del 2017 en el laboratorio de Tecnología de Alimentos y Análisis de Alimentos de la Escuela Profesional de Ingeniería en Industrias Alimentarias de la Universidad Nacional de Barranca, ubicado en el distrito y provincia de Barranca a una altitud de $56 \mathrm{msnm}$.

Para las pruebas experimentales se utilizaron 8 variedades de frijol común (Phaseolus vulgaris L.) sembradas en la campaña 20162017 (agosto - diciembre) en el campus agrícola de los Anitos. Cuando alcanzó el 
estado de madurez óptima $(12-14 \%$ de humedad en promedio), los granos se separaron de las plantas y se almacenaron a una temperatura ambiente en el Centro de Investigación de la Universidad Nacional de Barranca. Para la realización de los análisis correspondientes, las muestras se mantuvieron a temperatura ambiente.
Las muestras estuvieron conformadas por 2 $\mathrm{kg}$ de cada una de las 8 variedades de canario 2000, canario CIFAC, Laran mejorado, canario procedente de Huacaybamba, canario procedente de Ocros, Canario centenario, canario línea 4 y blanco Huanuqueño, todas cultivadas a las condiciones ambientales de la zona. Es pertinente mencionar que las semillas de las 3 primeras variedades fueron proporcionadas por el Instituto Nacional de Innovación Agraria de Chincha y Huaral.

Tabla 1. Variedades de frijol perteneciente a la colección de la Escuela de Ingeniería Agrónoma de la UNAB, 2016.

\begin{tabular}{|c|c|c|}
\hline $\mathbf{N}^{\circ}$ & $\begin{array}{l}\text { Color del } \\
\text { grano }\end{array}$ & Variedad \\
\hline $\mathrm{T}_{1}$ & $\begin{array}{l}\text { Amarillo } \\
\text { intenso }\end{array}$ & $\begin{array}{c}\text { Canario (procedencia Huacaybamba } \\
\text { Huánuco) }\end{array}$ \\
\hline $\mathrm{T}_{2}$ & $\begin{array}{l}\text { Amarillo } \\
\text { intenso }\end{array}$ & Canario (procedencia Ocros -Ancash) \\
\hline $\mathrm{T}_{3}$ & $\begin{array}{l}\text { Amarillo } \\
\text { intenso }\end{array}$ & Canario 2000 \\
\hline $\mathrm{T}_{4}$ & Amarillo claro & Canariocentenario \\
\hline $\mathrm{T}_{5}$ & Mostaza & Canario CIFAC \\
\hline $\mathrm{T}_{6}$ & Mostaza & Canariolínea4 \\
\hline $\mathrm{T}_{7}$ & Blanco & Laran mejorado(INIA) \\
\hline $\mathrm{T}_{8}$ & Blanco claro & Blanco Huanuqueño \\
\hline
\end{tabular}

La identificación de los granos por color es sumamente importante, pues según Ospina, (2002, citado por Cámara de Comercio de Bogotá 2015), menciona que es una de las características, más importantes de la determinación de la calidad en los granos. En el caso del frijol, este parámetro cambia de acuerdo a la variedad, pudiendo ser blanco, crema, rojo morado, marrón, rosado, negro, amarillo, entre otros.

Para la evaluación de los factores de calidad, se tomó las respectivas muestras que fueron llevadas a los laboratorios de Tecnología de Alimentos y Análisis de Alimentos de la Universidad Nacional de Barranca. Esta evaluación consiste en las características físicas y sensoriales las cuales son:
Masa de 100 granos de frijol: La masa como indicador del tamaño fue calculada según Mederos y Reynaldo (2007), lo cual determinó pesando 100 granos de frijol de cada variedad en una balanza analítica de precisión.

Volumen de 100 granos de frijol: Se determinó a partir del volumen de agua desplazado por una muestra de 100 granos de frijol de cada variedad, partiendo de un volumen preestablecido de agua, como lo establece Mederos y Reynaldo (2007).

Contenido de testa: Esta es la capa más externa del grano, que protege a los cotiledones de los factores externos; esta estructura ha sido relacionada con la acción de ser una cubierta protectora a la invasión de 
patógenos y desde el punto de vista sensorial con la textura del frijol cocido (Mederos y Reynaldo, 2007). Mencionado su importancia de la testa, se realizará la separación de la capa del grano, luego del remojo y posterior secada a $80^{\circ} \mathrm{C}$, hallando el porcentaje que la estructura representa respecto al grano.

Contenido de humedad: Se analizó el contenido de humedad en los granos, que convencionalmente se considera como el porcentaje de humedad, esto se obtiene del peso total del grano. Se empleó el indicador de humedad, para determinar la humedad del grano, lo cual está de acuerdo a lo establecido por la Norma Técnica Nicaragüense 16002 00 (2000).
Capacidad de absorción de agua: Es un parámetro conocido que puede ser empleado en el proceso de selección, para eliminar en generaciones tempranas aquellas variedades que absorben menor cantidad de agua, lo cual permite evaluar el grano de permeabilidad al agua del grano de frijol durante el remojo y detectar el fenómeno de testa dura, relacionado con prolongados tiempos de cocción (Mederos y Reynaldo, 2007).

Se determinó hallando la diferencia entre la masa inicial de 100 granos y la masa final, luego de ser remojadas en agua por 18 horas y cuantificadas mediante la siguiente fórmula establecida por Pérez et al. (2002).

$$
C A A=\frac{(M M D-M I M)}{M I M} \times 100
$$

Dónde:

MMD: Masa de la muestra después del remojo

MIM: Masa inicial de la muestra

Tiempo de cocción: Para la realización de dicho análisis se emplearon los siguientes materiales: una cocina, una olla de $2 \mathrm{~L}$ de capacidad y agua destilada, esta última fue llevada a ebullición y una vez alcanzado este punto se adicionó una muestra de 25 granos previamente remojados en agua durante 18 horas; durante el análisis se controlaron la ebullición y el volumen de agua hasta completar la cocción del grano, el tiempo de cocción es el tiempo requerido para que al menos el $90 \%$ de los granos de la muestra alcancen la cocción.

Evaluación sensorial: Se aplicó el método sensorial por estimación, por ordenamiento y escala hedónica de 9 puntos, utilizando panelistas entrenados, integrados por 6 miembros (chefs) de la Asociación de Hoteles, Restaurantes y Afines (AHORA- Barranca). Los atributos sensoriales evaluados fueron: la consistencia del caldo, el sabor, olor, color y la textura del grano cocido de las variedades como lo señala Leyva et al. (2010), cuyos datos fueron analizados mediante la prueba no paramétrica Kruskal-Wallis, procesados utilizando el programa Excel 2016 y Minitab 17.

\section{RESULTADOS}

La caracterización de las variedades de frijol estuvo referida a la masa y volumen de 100 granos, humedad del grano, capacidad de absorción de agua, porcentaje de testa, y tiempo de cocción, cuyos resultados se muestran en las siguientes Tablas. 
Tabla 2. Características físicas de 8 variedades de Phaseolus vulgaris $L$.

\begin{tabular}{|c|c|c|c|c|c|c|}
\hline Variedad & \multicolumn{2}{|c|}{ Masa de 100 granos $(\mathrm{g})$} & \multicolumn{2}{|c|}{$\begin{array}{l}\text { Volumen de } 100 \\
\text { granos }(\mathrm{ml})\end{array}$} & \multicolumn{2}{|c|}{$\begin{array}{l}\text { Humedad del } \\
\text { grano }(\%)\end{array}$} \\
\hline \multicolumn{7}{|l|}{ Canario (procedencia } \\
\hline $\begin{array}{l}\text { Huacaybamba - } \\
\text { Huánuco) }\end{array}$ & $45,0908 \pm 1,0344$ & $\mathrm{~F}$ & $36,33 \pm 0,58$ & de & $13,92 \pm 0,01$ & $a b$ \\
\hline $\begin{array}{l}\text { Canario (procedencia } \\
\text { Ocros - Ancash) }\end{array}$ & $55,0148 \pm 1,7518$ & bc & $37,67 \pm 0,58$ & $\mathrm{~cd}$ & $13,95 \pm 0,01$ & a \\
\hline Canario 2000 & $48,5925 \pm 0,8232$ & de & $33,67 \pm 0,58$ & $f$ & $13,40 \pm 0,02$ & \\
\hline Canario centenario & $44,1599 \pm 0,8317$ & $\mathrm{~F}$ & $33,50 \pm 0,50$ & $f$ & $13,89 \pm 0,02$ & $a b$ \\
\hline Canario CIFAC & $51,8997 \pm 1,2890$ & $\mathrm{~cd}$ & $38,67 \pm 0,58$ & C & $13,86 \pm 0,02$ & bc \\
\hline Canario Línea 4 & $46,6481 \pm 1,1022$ & ef & $35,67 \pm 0,58$ & e & $13,81 \pm 0,07$ & C \\
\hline $\begin{array}{l}\text { Canario Laran mejorado } \\
\text { (INIA) }\end{array}$ & $55,5182 \pm 1,6600$ & $\mathrm{~b}$ & $45,00 \pm 1,00$ & b & $13,85 \pm 0,01$ & bc \\
\hline Blanco Huanuqueño & $60,7623 \pm 0,7750$ & a & $47,33 \pm 0,58$ & a & $13,48 \pm 0,01$ & d \\
\hline \multirow{2}{*}{\multicolumn{7}{|c|}{ 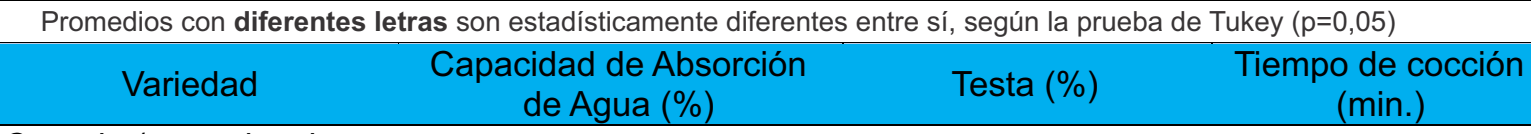 }} \\
\hline & & & & & & \\
\hline \multirow{3}{*}{$\begin{array}{l}\text { Canario (procedencia } \\
\text { Huacaybamba - } \\
\text { Huánuco) } \\
\text { Canario (procedencia } \\
\text { Ocros - Ancash) } \\
\text { Canario } 2000\end{array}$} & $100,0456 \pm 0,9472$ & a & \multicolumn{2}{|l|}{$6,1074 \pm 0,1211$} & $82 \pm 0$ & e \\
\hline & $96,9992 \pm 1,0896$ & bc & $6,5028 \pm 0,7523$ & $a b$ & $80 \pm 0$ & $g$ \\
\hline & $98,4863 \pm 0,3646$ & $a b$ & $6,3264 \pm 0,1443$ & abc & $79 \pm 0$ & $\mathrm{~h}$ \\
\hline Canario centenario & $95,8909 \pm 0,4645$ & $\mathrm{~cd}$ & $6,5490 \pm 0,0496$ & $a b$ & $83 \pm 0$ & $d$ \\
\hline Canario CIFAC & $96,9541 \pm 0,6445$ & bc & $5,9474 \pm 0,0322$ & $\mathrm{bc}$ & $85 \pm 0$ & $\mathrm{~b}$ \\
\hline Canario línea 4 & $97,8405 \pm 0,2214$ & $a b c$ & $5,9179 \pm 0,0243$ & bc & $84 \pm 0$ & C \\
\hline Laran mejorado (INIA) & $97,9334 \pm 1,6765$ & $a b c$ & $5,6200 \pm 0,1202$ & C & $85 \pm 0$ & a \\
\hline Blanco Huanuqueño & $93,5005 \pm 0,9001$ & $d$ & $7,0588 \pm 0,1345$ & a & $81 \pm 0$ & $f$ \\
\hline
\end{tabular}

Promedios con diferentes letras son estadísticamente diferentes entre sí, según la prueba de Tukey $(p=0,05)$

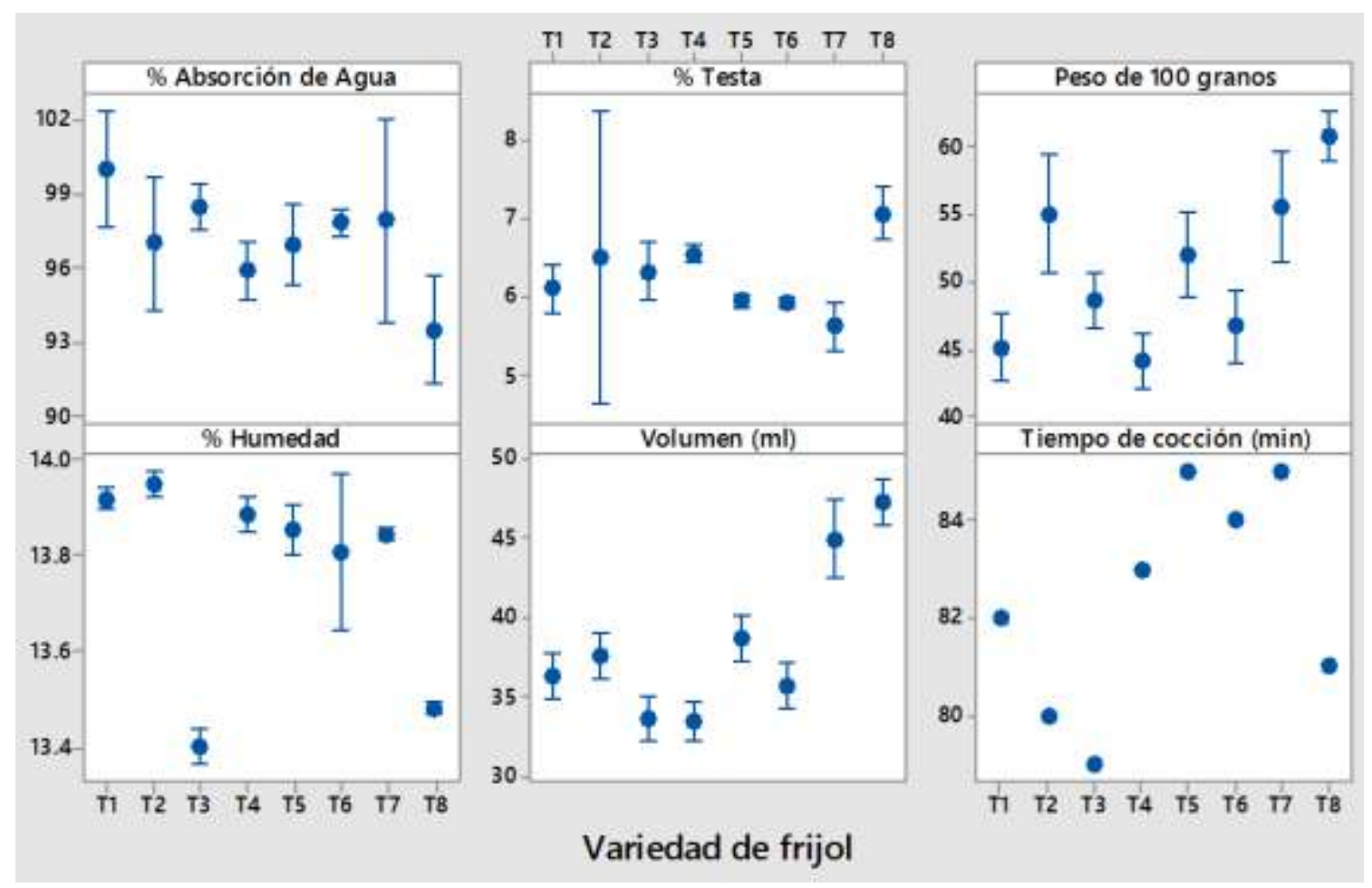

Figura 1. Intervalos de medición de características físicas del Phaseolus vulgaris $L$. 
Tabla 3. Resultados de la evaluación sensorial de frijoles cocidos por variedad.

\begin{tabular}{|c|c|c|c|c|c|c|c|}
\hline $\begin{array}{l}\text { Variedad } \\
\text { de frijol }\end{array}$ & $\begin{array}{l}\text { Consistencia } \\
\text { de caldo }\end{array}$ & Olor & Sabor & Textura & Color & Hedónica & Ordenamiento \\
\hline T1 & 9 & 8 & 8 & 7 & 10 & 8 & 7 \\
\hline T1 & 4 & 9 & 5 & 5 & 6 & 7 & 4 \\
\hline T1 & 10 & 10 & 9 & 8 & 10 & 7 & 4 \\
\hline $\mathrm{T} 1$ & 8 & 7 & 9 & 6 & 9 & 8 & 7 \\
\hline T1 & 5 & 3 & 6 & 5 & 6 & 6 & 4 \\
\hline T1 & 6 & 4 & 5 & 6 & 5 & 8 & 7 \\
\hline $\mathrm{T} 2$ & 6 & 9 & 10 & 9 & 10 & 7 & 4 \\
\hline T2 & 7 & 6 & 7 & 7 & 6 & 8 & 5 \\
\hline T2 & 6 & 8 & 7 & 6 & 5 & 5 & 3 \\
\hline T2 & 5 & 6 & 8 & 5 & 8 & 4 & 3 \\
\hline $\mathrm{T} 2$ & 3 & 5 & 5 & 6 & 5 & 8 & 8 \\
\hline T2 & 3 & 3 & 6 & 3 & 4 & 6 & 6 \\
\hline T3 & 5 & 9 & 8 & 9 & 10 & 7 & 3 \\
\hline T3 & 8 & 5 & 3 & 6 & 8 & 6 & 6 \\
\hline T3 & 4 & 7 & 7 & 5 & 6 & 8 & 2 \\
\hline T3 & 4 & 5 & 7 & 6 & 5 & 5 & 1 \\
\hline T3 & 3 & 6 & 4 & 5 & 6 & 7 & 3 \\
\hline T3 & 2 & 3 & 3 & 3 & 3 & 6 & 5 \\
\hline T4 & 9 & 9 & 10 & 10 & 10 & 9 & 8 \\
\hline $\mathrm{T} 4$ & 9 & 8 & 8 & 9 & 10 & 9 & 8 \\
\hline $\mathrm{T} 4$ & 7 & 6 & 9 & 10 & 10 & 9 & 8 \\
\hline $\mathrm{T} 4$ & 8 & 9 & 8 & 10 & 9 & 9 & 8 \\
\hline $\mathrm{T} 4$ & 8 & 7 & 8 & 6 & 6 & 9 & 7 \\
\hline $\mathrm{T} 4$ & 7 & 6 & 6 & 6 & 6 & 9 & 8 \\
\hline T5 & 5 & 8 & 8 & 7 & 10 & 7 & 5 \\
\hline T5 & 7 & 6 & 9 & 7 & 8 & 6 & 3 \\
\hline T5 & 5 & 4 & 6 & 4 & 7 & 6 & 6 \\
\hline T5 & 5 & 5 & 7 & 5 & 7 & 3 & 2 \\
\hline T5 & 4 & 4 & 5 & 4 & 5 & 6 & 6 \\
\hline T5 & 4 & 4 & 5 & 3 & 3 & 7 & 4 \\
\hline T6 & 6 & 9 & 10 & 9 & 10 & 8 & 6 \\
\hline T6 & 9 & 8 & 7 & 8 & 7 & 7 & 7 \\
\hline T6 & 4 & 5 & 7 & 5 & 8 & 7 & 7 \\
\hline T6 & 5 & 5 & 7 & 6 & 5 & 7 & 4 \\
\hline T6 & 4 & 5 & 6 & 5 & 5 & 7 & 2 \\
\hline T6 & 3 & 3 & 4 & 3 & 5 & 7 & 3 \\
\hline T7 & 7 & 8 & 8 & 7 & 8 & 6 & 2 \\
\hline $\mathrm{T} 7$ & 6 & 6 & 5 & 5 & 8 & 5 & 1 \\
\hline $\mathrm{T} 7$ & 4 & 6 & 6 & 5 & 5 & 6 & 1 \\
\hline $\mathrm{T} 7$ & 6 & 5 & 5 & 6 & 7 & 7 & 6 \\
\hline T7 & 6 & 3 & 6 & 6 & 6 & 5 & 1 \\
\hline $\mathrm{T} 7$ & 2 & 2 & 3 & 2 & 2 & 4 & 2 \\
\hline Т8 & 4 & 8 & 8 & 7 & 8 & 6 & 1 \\
\hline T8 & 3 & 4 & 5 & 5 & 8 & 6 & 2 \\
\hline T8 & 3 & 6 & 4 & 5 & 7 & 6 & 5 \\
\hline T8 & 6 & 6 & 6 & 7 & 8 & 6 & 5 \\
\hline T8 & 6 & 3 & 6 & 6 & 6 & 7 & 5 \\
\hline T8 & 1 & 3 & 3 & 2 & 2 & 5 & 1 \\
\hline
\end{tabular}


Tabla 4. Prueba de Kruskall-Walis para los atributos sensoriales

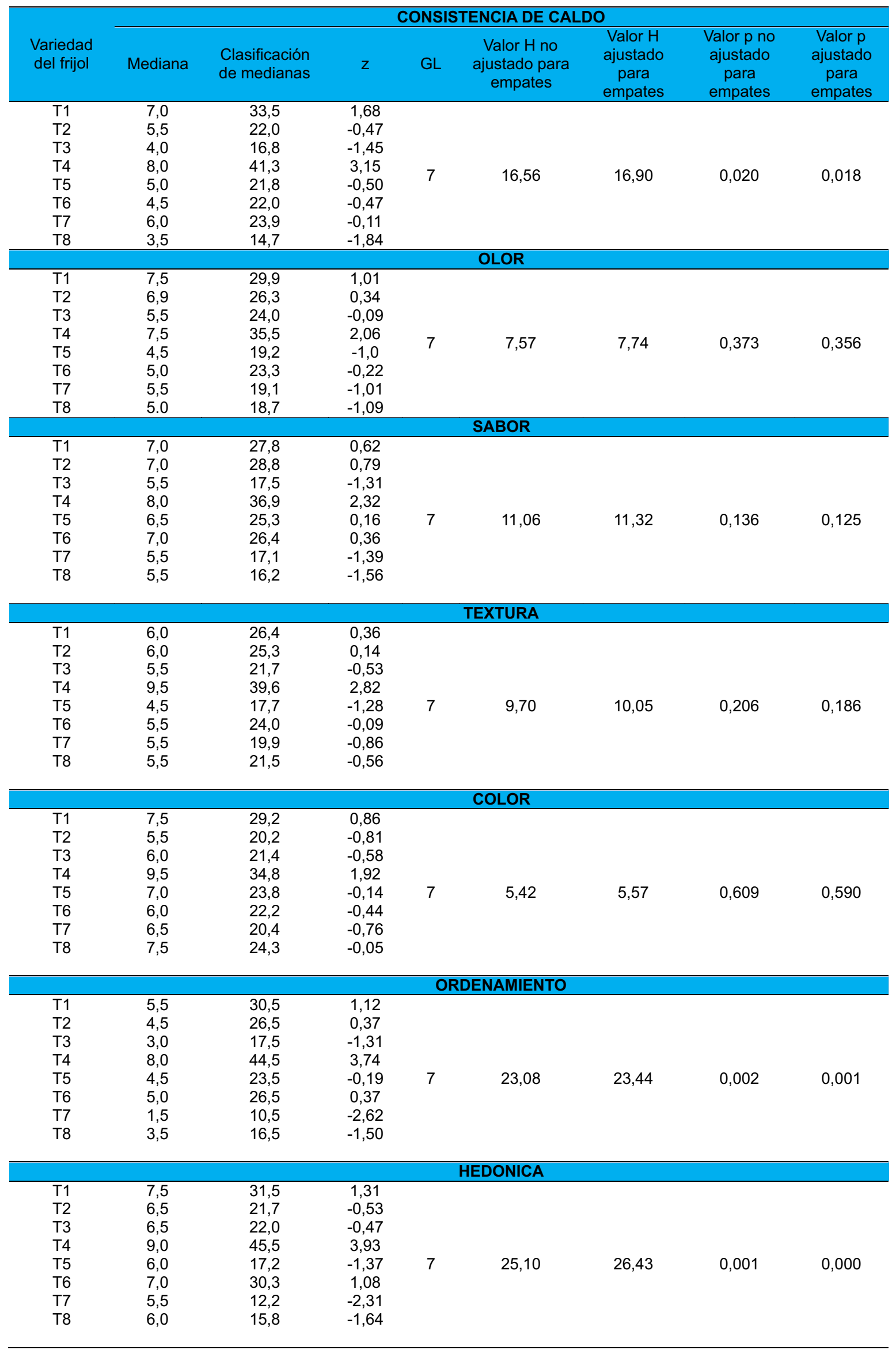

Si $p \leq 0,05$ las medianas no son todas iguales. 
Tabla 5. Estimación de atributos sensoriales de frijoles cocidos por variedad.

\begin{tabular}{|c|c|c|c|c|c|c|c|c|c|c|}
\hline Variedad & $\begin{array}{l}\text { Consis } \\
\text { del C }\end{array}$ & $\begin{array}{l}\text { encia } \\
\text { Ido }\end{array}$ & Olo & & Sab & & Textu & & Co & \\
\hline $\begin{array}{l}\text { Canario (procedencia } \\
\text { Huacaybamba - } \\
\text { Huánuco) }\end{array}$ & 7,000 & $a b$ & 6,830 & a & 7,000 & a & 6,167 & $a b$ & 7,667 & a \\
\hline $\begin{array}{l}\text { Canario (procedencia } \\
\text { Ocros - Ancash) }\end{array}$ & 5,000 & $a b$ & 6,167 & a & 7,167 & a & 6,000 & $a b$ & 6,333 & a \\
\hline Canario 2000 & 4,333 & $\mathrm{~b}$ & 5,833 & a & 5,333 & a & 5,667 & $a b$ & 6,333 & a \\
\hline Canario centenario & 8,000 & a & 7,500 & a & 8,167 & a & 8,5000 & a & 8,500 & a \\
\hline Canario CIFAC & 5,000 & $a b$ & 5,167 & a & 6,667 & a & 5,000 & $\mathrm{~b}$ & 6,667 & a \\
\hline Canario línea 4 & 5,167 & $a b$ & 5,833 & a & 6,833 & a & 6,000 & $a b$ & 6,667 & a \\
\hline $\begin{array}{l}\text { Laran mejorado } \\
\text { (INIA) }\end{array}$ & 5,167 & $a b$ & 5,000 & a & 5,500 & a & 5,167 & $a b$ & 6,000 & a \\
\hline Blanco huanuqueño & 3,833 & $b$ & 5,000 & a & 5,333 & a & 5,333 & $a b$ & 6,500 & a \\
\hline
\end{tabular}

Promedios con diferentes letras $(\mathbf{a}, \mathbf{b})$ son estadísticamente diferentes entre sí, según la prueba de Tukey $(p=0,05)$

Tabla 6. Estimación de atributos sensoriales por ordenamiento y escala hedónica

\begin{tabular}{|c|c|c|c|c|}
\hline Variedad & \multicolumn{2}{|c|}{ Ordenamiento } & \multicolumn{2}{|c|}{ Escala Hedónica } \\
\hline Canario centenario & 7,833 & a & 9,000 & a \\
\hline $\begin{array}{l}\text { Canario (procedencia } \\
\text { Huacaybamba - } \\
\text { Huánuco) }\end{array}$ & 5,500 & $a b$ & 7,333 & $a b$ \\
\hline Canario línea 4 & 4,833 & $a b c$ & 7,167 & $a b$ \\
\hline $\begin{array}{l}\text { Canario (procedencia } \\
\text { Ocros - Ancash) }\end{array}$ & 4,833 & $a b c$ & 6,333 & b \\
\hline Canario 2000 & 3,333 & bc & 6,500 & b \\
\hline Blanco huanuqueño & 3,167 & $\mathrm{bc}$ & 6,000 & b \\
\hline Canario CIFAC & 4,333 & $\mathrm{bc}$ & 5,833 & b \\
\hline Laran mejorado (INIA) & 2,167 & $\mathrm{c}$ & 5,500 & b \\
\hline
\end{tabular}

\section{DISCUSIÓN}

La producción agrícola del frijol, es una de las principales actividades económicas en la provincia de Barranca; puesto que cada vez más aumenta áreas de siembra con el fin de obtener mayor ganancia. Sin embargo, se debe tener en cuenta las características físicas y sensoriales del grano, como una condición de calidad del producto.

El análisis de masa y volumen de 100 granos, como indicadores de tamaño, determina la existencia de diferencias estadísticas entre las variedades de frijol, lo que significa que existe variabilidad en los resultados; siendo Blanco Huanuqueño el mayor. La masa y volumen de 100 granos, mantienen una relación directa, observándose a mayor peso, mayor volumen; estos resultados son similares a los encontrados en investigaciones realizadas en genotipos de la especie Phaseolus vulgaris L., por Mederos y Reynaldo (2007). Las variedades con granos de color amarillo y blanco presentan comportamientos similares. El blanco huanuqueño es la variedad de mayor tamaño, presenta mayor masa y volumen (Tabla 2 y Figura 1), seguida por el Laran mejorado junto con el canario procedente de Ocros que tienen valores muy próximos y estadísticamente son similares. El Canario centenario presenta menor valor de masa y volumen, que estadísticamente es similar al canario procedente de Huacaybamba y canario línea 4. Los granos de un mismo color presentan diferencias de tamaño; sin embargo, estas se hacen notablemente marcadas entre los granos de color amarillo, mientras que las blancas presentan masa y volumen más homogéneos. 
Al realizar el análisis del contenido de humedad en los granos de las 8 variedades en estudio, se evidenciaron diferencias en los niveles, aun cuando fueron almacenadas en igualdad de condiciones. De forma general, el contenido de humedad en las 8 variedades osciló de un $13,40 \%$ a $13,95 \%$, que muestra una variación significativa de los resultado del buen manejo en el secado previo al almacenamiento, esto se relaciona con De Oliveira (2002), menciona que la humedad es un factor a tener en cuenta al momento de analizar los granos, debido a que altos niveles de humedad y temperatura (mayores que 25 $\left.{ }^{\circ} \mathrm{C}\right)$ pueden acelerar el deterioro gradual, irreversible y acumulativo de estos durante el almacenamiento. También Mora (1980) al realizar estudios sobre el efecto de la humedad y la temperatura sobre el endurecimiento del frijol (Phaseolus vulgaris L), observó que los granos pueden ser almacenados a una temperatura de $25^{\circ} \mathrm{C}$ y un $15,4 \%$ de humedad, sin que sean detectados problemas de endurecimiento, por lo que teniendo en cuenta el periodo de almacenamiento, la temperatura y el nivel de humedad del grano en los materiales de estudio, estos presentan un adecuado índice de humedad que no debe influir en el resultado de los análisis. El canario procedente de Ocros presentó mayor contenido de humedad, que mantiene una similitud estadística con el canario procedente de Huacaybamba. Además, el Canario 2000 es la variedad con menor contenido de humedad.

De forma general, el porcentaje de testa se presentó como un indicador de baja variabilidad, es decir una significativa homogeneidad encontradas entre las variedades con granos de color amarillo. Dicho porcentaje en las variedades de frijol de color amarillo muestra leves diferencias entre ellas. Estos valores oscilan entre un 5,62\% (Laran mejorado) y un $7,06 \%$, propio del Blanco Huanuqueño. Los resultados demuestran que, a mayor peso de grano, mayor es el porcentaje de testa.

Al efectuar el análisis de capacidad de absorción de agua en las variedades de granos de color amarillo y blanco, se obtuvieron datos ligeramente diferentes entre ambos grupos, lo cual indica que tienen una significativa variación. La comparación entre la capacidad de absorción de agua y el porcentaje de testa, la primera no depende de la segunda, lo cual se refleja en el hecho de que los granos de color blanco de manera general presentan un valor relativamente superior en el contenido de testa y menor capacidad de absorción de agua. El Laran mejorado presenta un contenido de testa inferior a la del Canario línea 4 y también valores bajos de capacidad de absorción de agua, por lo que un aumento del contenido de testa no repercute en la capacidad de absorción de agua de la variedad; esto está en total concordancia con lo informado por Egley (1989), citado por Mederos y Reynaldo (2007), que afirma la impermeabilidad de la testa no solo está dada por el porcentaje de esta, ya que otros compuestos o estructuras intrínsecas pudieran afectar la inhibición. Otros autores como Jacinto et al. (2002), manifiestan que la capacidad de absorción de agua es un factor que, en algunos casos, es indicador del tiempo de cocción. Por los resultados se puede afirmar que no existe una relación directa entre capacidad de absorción de agua y el porcentaje de la testa de las variedades estudiadas.

Constituye uno de los parámetros de calidad más importantes en el grano de fríjol, puesto que Mederos y Reynaldo (2007), menciona que los tiempos cortos constituyen una característica que ha sido seleccionada durante los procesos de domesticación y es considerado un atributo de la calidad sensorial. Asimismo, Mederos y Reynaldo (2007), menciona los tiempos de cocción pueden prolongarse por dos procesos diferentes relacionados con la absorción de agua por parte de las semillas: el primero se denomina "testa dura" y se manifiesta cuando las semillas no absorben suficiente agua durante la cocción y por tal razón, al ser cocinadas no se ablandan. Esto puede estar dado por la baja permeabilidad de la testa al agua. El segundo proceso se denomina "difícil de cocinar" y se manifiesta en aquellas semillas que absorben suficiente agua, pero fallan al ablandarse luego del remojo y posterior tiempo de cocción. Este es un indicador de calidad sumamente importante tanto para los productores como para consumidores, ya que los tiempos relativamente cortos ahorran combustible y tiempo, como lo señalan Mederos y Reynaldo 
(2007). Tapia (1985), encontraron que factores como tamaño de grano, brillo y porcentaje de testa en el grano de frijol son determinantes en el tiempo de cocción, asimismo señalan que el remojar los frijoles por 16 horas antes de la cocción suaviza el grano y disminuye el tiempo de cocción.

Al realizar una comparación entre los tiempos de cocción de las variedades analizadas, se evidencian diferencias significativas entre las variedades con granos de un mismo color. Los resultados en ambos grupos muestran una gran variabilidad de los resultados. Las variedades de frijol presentaron tiempos de cocción entre 79 y 85 minutos. De acuerdo al análisis del tiempo de cocción, el Canario 2000 presenta menor tiempo de cocción, mientras que el laran mejorado y Canario CIFAC presentan los mayores tiempos de cocción.

Las muestras previamente preparadas fueron sometidas a un grupo de panelistas entrenados, considerando una escala de 1 a 10, cuyos resultados muestran ligeras diferencias. De acuerdo a los resultados de los atributos sensoriales en relación a la consistencia del caldo, según la prueba no paramétrica de Kruskall - Wallis las variedades de frijol presentan diferencias estadísticamente significativas $(p \leq 0,05)$, asimismo por la prueba de Tukey, el canario 2000 y blanco huanuqueño presentan características similares, en comparación a las demás variedades. Asimismo, el Canario Centenario presenta mejor consistencia. Estos resultados coinciden con lo reportado por Leyva et al. (2010), que no encontró diferencia significativa $(p=0,9)$ en el porcentaje de participantes que clasificaron (en una escala de uno a cuatro) la consistencia del caldo.

En relación al olor, sabor, color y textura, todas las variedades presentan características similares, como lo evidencia la prueba de Kruskall - Wallis $(p>0,05)$, no existiendo diferencias significativas. En cuanto a la textura según la prueba de Tukey, la única variedad que es diferente a las demás es el canario CIFAC, que no presenta buena textura desde el punto de vista sensorial. Estos resultados coinciden con lo reportado por Leyva et al. (2010), en cuyo estudio no hubo diferencia significativa $(P=0,9)$ en el porcentaje de participantes que clasificaron (en una escala de uno a cuatro) el sabor y la textura del grano entre las variedades.

En cuanto a la buena textura se aprecia el canario centenario con mayor preferencia. De acuerdo a la prueba de ordenamiento el canario centenario presenta mejores condiciones de aceptabilidad por parte de los panelistas, por tener mayor puntaje promedio; pero que estadísticamente es similar a las variedades canario procedente de Huacaybamba, canario procedente de Ocros, y el canario línea 4. Estos resultados coinciden con lo encontrado por Carrillo (2011), que realizaron un trabajo de aceptación de 3 líneas de frijol en la que no hubo diferencia estadísticamente significativa en el puntaje asignado a la aceptabilidad general y a los atributos olor, color, sabor y textura $(p>0,05)$.

Para preferencia, no se encontró una diferencia en el porcentaje de personas que preferían cada una de las 4 muestras, concluyendo que al no haber diferencia de aceptabilidad ni preferencia entre las líneas evaluadas, se esperaría obtener frijoles con mejores características nutricionales.

Los resultados de aplicación de escala hedónica de 9 puntos, evidencia que existe diferencias significativas entre las variedades $(p \leq 0,05)$, sin embargo la prueba de Tukey demuestra que el canario centenario es la que mejor gusto presenta para el consumidor, pero que estadísticamente es similar a las variedades canario procedente de Huacaybamba y canario línea 4.

A partir de las dos pruebas de ordenamiento y de escala hedónica se concluye que el Canario centenario alcanza la mayor puntuación, siendo la variedad con mejor aceptación desde el punto de vista sensorial.

\section{AGRADECIMIENTO}

Agradecimiento especial a la Universidad Nacional de Barranca, que financió el estudio a través del proyecto "Evaluación de variedades mejoradas de Phaseolus vulgaris $L$. frijol canario, para incrementar su rendimiento en la provincia de Barranca". 


\section{REFERENCIAS BIBLIOGRÁFICAS}

Cárdenas, H., Gómez, C., Díaz J. \& Camarena, F. (2000). Evaluación de la calidad de la proteína de 4 variedades mejoradas de frijol. Revista Cubana de Alimentación y Nutrición. 14 (1), 7-22.

Carmona, R. (2005). Efecto del tipo de remojo en la digestibilidad del almidón en frijol cocido (tesis de postgrado). Instituto Politécnico Nacional. Yautepec, Morelos - México.

Carrillo, P., Chow, Z., Cuadra, S., Brenes, D. \& Pachón, Ch. (2011). Aceptación de tres líneas de fríjol (Phaseolus vulgaris L.) mejorado nutricionalmente por los consumidores nicaragüenses. Perspectivas en Nutrición Humana. 13(2), 179.

De Oliveira, A., Patto, C. \& Duarte, A. (2002). Efeitos da época de colheita e do tempo de armazenamento no escurecimento do tegumento do feijão (Phaseolus vulgaris). Ciênc. Agrotec. Lavras. 26(3), 550-558.

Dirección General de Industrias Básicas. (2012). Análisis de la cadena de valor del frijol. Secretaría de Economía. México.

INEI. (2009). Consumo de Alimentos y Bebidas. Informe estadístico. Lima-Perú.

Jacinto, C. et al. (2002). Caracterización de una población de líneas endogámicas de frijol común por su calidad de cocción y algunos componentes nutrimentales. Agrociencia. 36, 451-459.

Jacinto, C. \& Campos A. (1993). Efecto de la cocción sobre algunas características nutricionales del frijol. Agronomía Mesoamericana. 4, 42-47. Recuperado d http://www.mag.go.cr/rev_meso/v04n01 042.pdf

Leyva, R., Pachón, H., Chaveco, O., Permuy, N., Ferraz, Y., Caballero, N., \& García, E. (2010). Evaluación sensorial de frijol (Phaseolus vulgaris L.) mejorado nutricionalmente en dos comunidades cubanas. Agronomía Mesoamericana.
21(2), 281-288. Recuperado de file:///C:/Users/Administrador/Downloads 14890-7211-1-PB.pdf

Mederos, Y. \& Reynaldo, I. (2007). Determinación de indicadores de calidad en 11 genotipos de la especie Phaseolus vulgaris, L. Cultivos Tropicales. 28(4), 515 6. R e c u p e r a d o d e http://www.redalyc.org/pdf/1932/193217 894007.pdf

Mora, M. (1980). Efecto de la humedad y temperatura en el endurecimiento de frijol (Phaseolus vulgaris. L.) almacenado durante seis meses. Agronomía. 4(2), 195-197.

Norma Técnica Nicaragüense (2000). Norma Técnica Nicaragüense 16002-00 para frijol en grano. Recuperado de extwprlegs 1 .fao.org/docs/texts/nic45922. doc

Ospina, J. (2002). Características físico mecanizadas y análisis de calidad en granos. Publicaciones Universidad Nacional de Colombia.

Pérez, P., Esquivel, G., Rosales, R., \& Acosta, J. (2002). Caracterización física, culinaria y nutricional de frijol del altiplano subhúmedo de México. Archivos Latinoamericanos de Nutrición. 52(2). 172-180.

Tapia, B.; López, S. \& Estrada, B. (1985). Evaluación de un método para reducir el tiempo de cocción en frijol común. XXXI Reunión Anual del PCCMCA, San Pedro Sula, Honduras.

Ulloa, J., Ulloa P., Ramírez, J. \& Ulloa, B. (2011). El frijol (Phaseolus vulgaris): su importancia nutricional y como fuente de fitoquímicos. Fuente. 3(8). 5-9. $R$ e c u p e r a d o d e http://fuente.uan.edu.mx/publicaciones/0 3-08/1.pdf

\section{Correo electrónico:}

njamanca@unab.edu.pe

Revisión de Pares:

Recibido: 27 - 04 - 2018

Aceptado: 22 - 06 - 2018 